\title{
Increased expression of toxoplasma gondii gra I suppresses host cell apoptosis
}

\begin{abstract}
Suppression of host cell apoptosis enable Toxoplasma gondii to proliferate, but the mechanism is not well understood. We explore the relationship between the expression of $T$. gondii dense granule protein 1 (GRA1) and host HeLa cell apoptosis. We inserted the $T$. gondii gra1 coding gene into a pET32a vector and produced recombinant GRA1 (rGRA1) in $E$. coli Rosetta strain. We then immunized rabbits with rGRA1 to produce anti-GRA1 serum and infected HeLa cells with $T$. gondii tachyzoites. We determined the expression of T. gondii GRA1 by Western blotting with anti-GRA1 serum and determined apoptosis of HeLa cells via the Annexin V-FITC/PI method. All the experiments were repeated for three times in the same condition. The expression of GRA1 decreased gradually after $T$. gondii infection. The rate of HeLa cell apoptosis increased more rapidly in the infected cells than in the uninfected cells. Our results suggest that the suppression of host cell apoptosis is related to the expression of $T$. gondii GRA1 expression.
\end{abstract}

Keywords: toxoplasma gondii, GRA1, apoptosis, ROPs, GRAs, STAT
Volume 4 Issue 5 - 2017

\author{
Liang Wu,' Jin Shen,' Chenyu Tang, ${ }^{2}$ Xugan \\ Jiang,' Jiajian Wang, ${ }^{2}$ Xiaoling Jin, ${ }^{2}$ Jinbo Qiu, ${ }^{2}$ \\ Shengxia Chen,' Jianping $\mathrm{Cao}^{3}$ \\ 'School of Medicine, Jiangsu University, China \\ 2Jingjiang College of Jiangsu University, China \\ ${ }^{3}$ National Institute of Parasitic Diseases, China \\ "Liang Wu and Jin Shen contribute equally to this work
}

Correspondence: Shengxia Chen, School of Medicine, Jiangsu University, Zhenjiang 21 20 I3, China, Tel +86 I39528I5350, Email chensxia@ujs.edu.cn

Received: April 25, 2017| Published: May 16, 2017

\section{Introduction}

Toxoplasma gondii is an obligate intracellular protozoon parasite. In immunocompromised hosts, such as AIDS patients, it becomes an opportunistic pathogen causing severe toxoplasmosis. ${ }^{1}$ Apoptosis is a common biological phenomenon that removes damaged or unwanted cells during development, ensuring tissue homeostasis in multi-cellular organisms. ${ }^{2}$ Cell apoptosis also plays a major role in innate and adaptive immune defense by controlling and eradicating invasive pathogens. ${ }^{3} T$. gondii, however, can actively invade any nucleated host cell and, by down-regulating apoptosis in the cell, can evaded elimination by the host's defense system. ${ }^{4}$ Although the mechanism of this down-regulation is not yet understood, it is known that $T$. gondii secreted proteins including rhoptries (ROPs) and dense granule proteins (GRAs) play an important role in this regulation. ${ }^{5}$ Many studies have examined the function of ROPs, but few have studies the function of GRAs. ${ }^{6}$ In this study, we investigate variation in the expression of GRA1 and in the rate of cell apoptosis during T. gondii invasion into HeLa cells.

\section{Material and methods}

\section{Ethics statement}

All animal experiment in the manuscript were reviewed in advance by the Laboratory Animal Management Committee of Jiangsu University and also met the guidelines of the National Institutes of Health Guide for the Care and Use of Laboratory Animals.

\section{Cell culture and T. gondii}

The HeLa cell line was stored in liquid nitrogen in our laboratory and cultured in DMEM medium (HyClone, Argentina) and incubated with $10 \%$ fetal bovine serum at $37^{\circ} \mathrm{C}$ and at $5 \% \mathrm{CO}_{2}$ (Tianhang Biological Technology Co., Ltd, China). The cells were stripped from the cell culture flask with a $0.25 \%$ trypsin (GBICO, USA) and $0.02 \%$ EDTA-Na2 solution. The passage operation was repeated every 2-3days. T. gondii RH strain tachyzoites were maintained in HeLa cells. Before infection with $T$. gondii tachyzoites for $12 \mathrm{~h}$, the HeLa cell medium was changed to a DMEM medium with $2 \%$ fatal bovine serum. The T. gondii RH strain tachyzoites were added into the culture medium and this was changed to new fresh DMEM medium (with 2\% fetal bovine serum) 4hours after infection. After HeLa cell lysis, the tachyzoites were collected by centrifugation and purified using a $3-\mathrm{mm}$ filter membrane. ${ }^{7}$

\section{Cloning of the GRAI gene and anti-GRAI serum pro- duction}

The purified tachyzoites were used for total RNA extraction performed using the RNaEXTM Total RNA Isolation Kit according to the manufacturer's instructions (Generay Biotech Co., Ltd. China). The GRA1 gene used for His-GRA1 recombinant protein production was amplified from cDNA by PCR assay. The GRA1 primers 5'- CGGATCCCCGAAGGCGGCGACAACCAG-3' (sense) and 5'- GAATTCTACTCTCTCTCTCCTGTTAGGAACCCAATGTC-3' (antisense) yielded a 495-nucleotide product specific to the GRA1 coding gene (GenBank No. HM067753.1). The PCR product was digested with BamHI and EcoRI, ligated into a pET32a vector using T4 DNA ligase (TAKARA, Dalian, China), and transformed into an E. coli Rosetta strain. The right directional clone was confirmed by sequencing. The His-GRA1 recombinant protein was expressed in E. coli Rosetta strain that had been induced at $30^{\circ} \mathrm{C}$ for $10 \mathrm{~h}$ with $0.4 \mathrm{mM}$ isopropyl- $\beta$-D-thiogalactopyranoside (IPTG). The bacterial pellets were re-suspended in lysis buffer (Tris-HCl 10mM [pH 8.0], NaH2PO4 100mM, Urea $8 \mathrm{M})$. Cellular debris was removed by centrifugation at $16,000 \mathrm{~g}$ for 30min. The cell-free extract was used for rHis-ACP purification by Ni-NTA Agarose (QIAGEN, Hilden, Germany) according to the manufacturer's instructions. This His-ACP recombinant protein was identified by SDS-polyacrylamide gel electrophoresis staining with Coomassie blue.

The specific anti-serum of GRA1 was obtained from New Zealand rabbits (male, $3 \mathrm{~kg}$, Laboratory Animal center of Jiangsu University) by three subcutaneous immunizations. The first injection comprised 
1mg of recombinant GRA1 in Freund's complete adjuvant, with two further injections also with Freund's incomplete adjuvant administered at three week intervals. The rabbit was then bled and the specific anti-serum collected for the Western blotting assay.

\section{Western blotting assay}

Freshly released tachyzoites were boiled in SDS-PAGE sample buffer and separated on $12 \%$ polyacrylamide gels utilizing the Laemmli discontinuous buffer system. ${ }^{8}$ Proteins were transferred to nitrocellulose membranes (BOSTER, China), and the nitrocellulose strips were saturated for $1 \mathrm{~h}$ in $5 \%$ non-fat milk in $15 \mathrm{mM}$ Tris- $\mathrm{HCl}$ (pH 8.0), $150 \mathrm{mM} \mathrm{NaCl}$, and $0.05 \%$ Tween 20 (TNT). They were then incubated with anti-GRA1 serum diluted 1: 2,000 in TNT for 1h. After washing, the strips were incubated with goat anti-rabbit IgG conjugated to HRP (BOSTER, China) and detected using the ECL (BOSTER, China) plus Western blotting system.

\section{T. gondii GRAI expression}

The parasites $\left(5 \times 10^{6} /\right.$ well $)$ were added to confluent HeLa cell monolayers in a 6-well plate (ratio of cells to parasites equals one). After infection for $1 \mathrm{~h}$, all the infected HeLa cells were completely washed with PBS to eliminate parasites that had not invaded cells. We used anti-GRA1 serum and anti- $T$. gondii actin mAb (in mouse ascitic fluids, obtained from Professor Dominique of the University of Geneva, Switzerland) to determine the expression of GRA1 during infection.

\section{Analysis of apoptosis}

The parasites $\left(5 \times 10^{6}\right.$ per well) were added to confluent HeLa cell monolayers in a 6-well plate (at a cell to parasite ratio equal to one). One hour after infection, all infected HeLa cells were completely washed with PBS to eliminate parasites that had not invaded cells. After cell detachment, HeLa cells were stained with Annexin V using the Annexin V-FITC/PI kit (Vazyme, Nanjing, China). Apoptosis cells were identified and quantified by flow cytometry (FACSCalibur, BD Company, USA). Briefly, HeLa cells $\left(1 \times 10^{6}\right.$ per well $)$ were washed in PBS and incubated with $1 \times$ binding buffer, propidium iodide $(\mathrm{PI})$ and Annexin V-FITC for $30 \mathrm{~min}$ at room temperature. The apoptotic cells were analyzed on a fluorescence-activated cell sorter.

\section{Statistical analyses}

Statistical analyses were performed in SPSS. Student's $t$ tests were performed under the assumption of equal variance and using a two-tailed test, where $\mathrm{P} \leq 0.05$ was considered significant.

\section{Results}

\section{Expression of rGRAI and anti-GRAI serum produc- tion}

Recombinant plasmids containing GRA1 gene were confirmed by BamHI/EcoRI restriction enzyme analysis. The band corresponding to the $495 \mathrm{bp}$ product was visualized on a $1.5 \%$ of agarose gel (Figure 1). As shown in the SDS-PAGE gel, the rGRA1 expression was induced by IPTG and its molecular weight was about $45 \mathrm{kDa}$. This is consistent with the expected molecular mass given that the expression vector produced a recombinant protein fused to a $20 \mathrm{kDa}$ His-thioredoxin tag (Figure 2). In the Western blotting assay, the GRA1 (25kDa) in tachyzoite lysis could be recognized by the rabbit anti-GRA1 serum, while the rabbit anti-GRA1 serum did not react with the HeLa cell lysis (Figure 3 ).

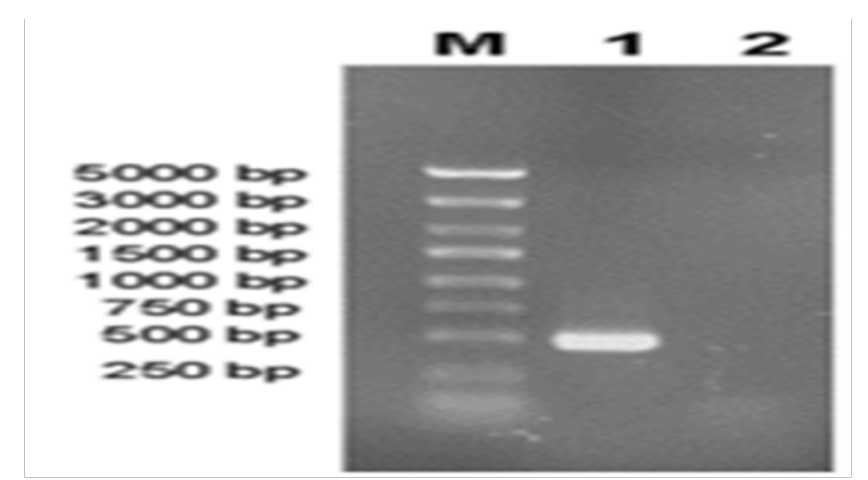

Figure I The GRAI gene product amplified by RT-PCR from T. gondii (RH strain).

M, molecular weight marker; Lane I, GRAI gene; Lane 2, negative control.

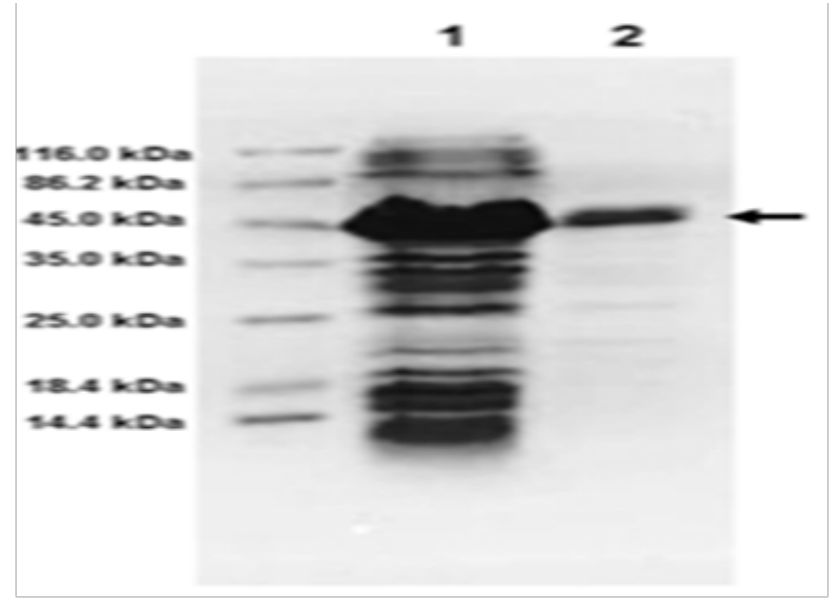

Figure 2 SDS-PAGE analysis of rGRAI expressed in E. coli.

The E. coli lysates were electrophoresed on I2\% SDS-PAGE and stained with Coomassie brilliant blue R-250. M: molecular weight marker; Lane I: IPTGinduced E. coli with pET-32a; Lane 2: Purified rGRAI. The rGRAI fused with His-thioredoxin tag $(45 \mathrm{kDa})$ is shown with an arrow.

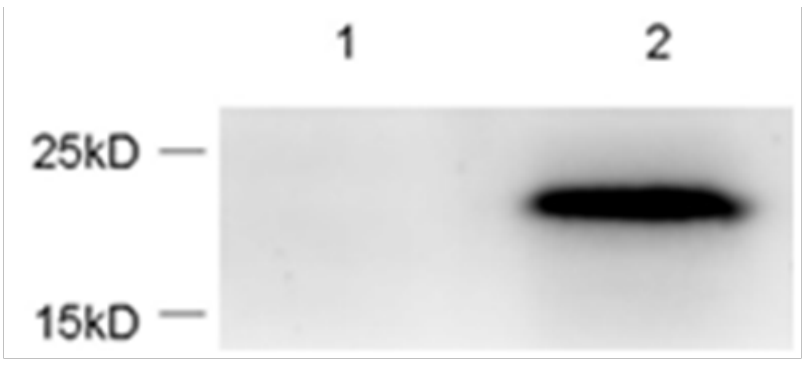

Figure 3 Western blotting analysis of expressed rGRAI.

Lane I: HeLa cells; Lane 2: T. gondii.

\section{GRAI expression in T. gondii infection}

The expression of GRA1 decreased gradually following the infection of HeLa cells by $T$. gondii. One hour post-infection the expression of GRA1 peaked and then decreased rapidly to its lowest level at 36 post-infection (Figure 4).

\section{HeLa cell apoptosis}

The rate of HeLa cell apoptosis increased with time for both infected and uninfected cells, but did so more rapidly in the uninfected 
group. At eight hours post-infection, there was no significant difference in apoptosis rates between infected and uninfected individuals. However, by 12 hours post-infection, the rate of apoptosis of uninfected cells had increased dramatically. At 36hours post-infection, we observed the greatest difference in the rate of apoptosis in these two groups (Figure 5).

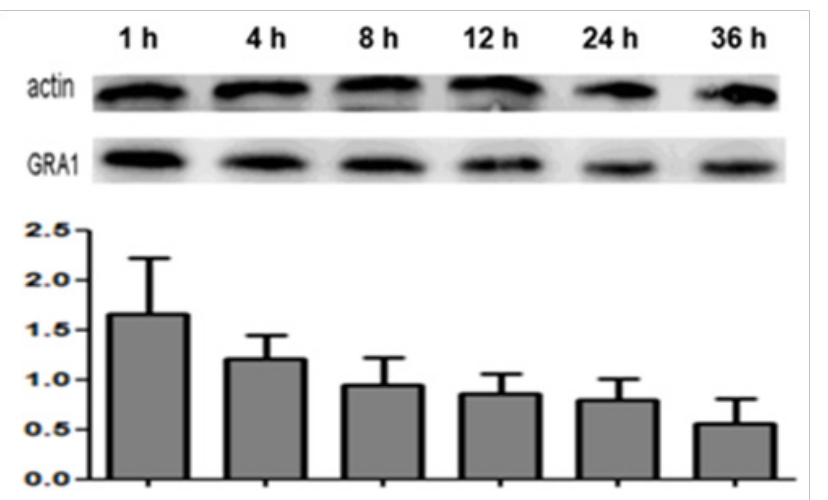

Figure 4 Western blotting analysis of T. gondii GRAI expression over 36h.

Actin: T. gondii actin expression; GRAI: T. gondii GRA I expression.

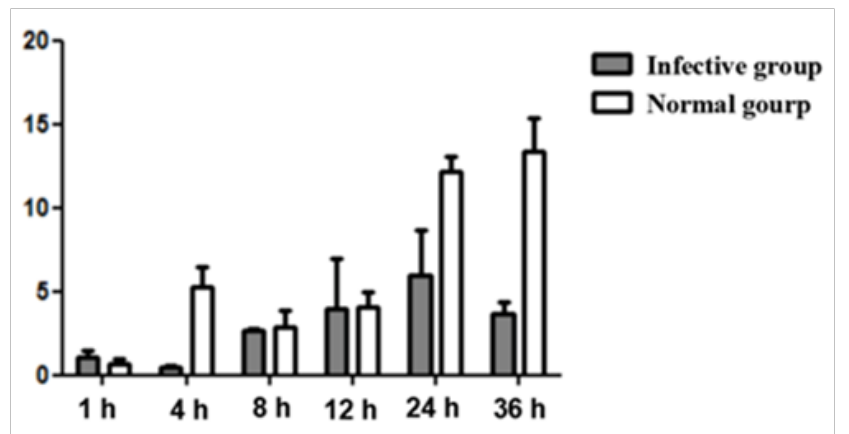

Figure 5 Rates of apoptosis of HeLa cells infected with T. gondii and in uninfected cells over a 36-hour period.

\section{Discussion}

The obligate intracellular protozoan $T$. gondii has evolved an intimate relationship with its host that extends to the cellular and molecular levels. ${ }^{9}$ This pathogen requires an appropriate host cell environment for its replication and is able to modify its host cell functions. ${ }^{10}$ Intuitively, the active modification of host cell growth must be a complex process, as host cells are not inherently programmed to provide an environment conducive to pathogens. ${ }^{11,12}$ Host cells have evolved primary lines of defense as countermeasures to pathogen invasion, establishment, and replication. ${ }^{13,14}$ Defenses to limit pathogen growth include apoptosis, reactive oxygen and nitrogen intermediates. ${ }^{15}$ Previously, we reported that $T$. gondii infection suppressed apoptosis of $\mathrm{HeLa}$ cells for 36 hours post-infection in uninfected cells and in cells treated with actinomycin D. ${ }^{16}$ T. gondii uses a mixture of specialized parasite secreted proteins, including ROPs, GRAs and MICs to invade cells. ${ }^{17,18}$ Many of these parasite secreted proteins are anchored on the parasitophorous vacuole membrane (PVM) and can interact with the host cells directly. The kinase activity ROPs, such as ROP $2,{ }^{19}$ ROP $16^{20}$ and ROP $18,{ }^{21}$ have been exhaustively studied and shown to down-regulate host cell apoptosis. ${ }^{22}$ Moreover, kinase activity ROPs can affect the activation of signal transducer and activator of transcription (STAT) signaling pathways, leading to a downstream modulation of the secretion of IL-12 in host cells..$^{23,24}$
GRA1 belongs to a dense granule protein family consisting of 12 distinct polypeptides that are stored within dense granules of $T$. gondii. ${ }^{25}$ During cell invasion, GRA1 was exocytosed into the parasitophorous vacuole (PV) and associated with PVM. Unlike T. gondii ROPs, the role of GRAs is not yet clearly understood. Indeed, GRA1 could activate TGF- $\beta$ transcription through phosphorylation and activate apoptosis in monocytes. ${ }^{26}$ The role of GRAl in human cell apoptosis has rarely been studied. In this study, we showed that the expression of GRA1 decreased gradually 36 hours post-infection, while HeLa cell apoptosis increased more rapidly in infected cells compared to uninfected cells. These findings suggest that GRA1 suppresses HeLa cell apoptosis, in a manner similar to ROPs.

\section{Conclusion}

We suggest that GRA1 is anchored in the PVM and along with other secreted proteins, such as ROP2, could down-regular human cell apoptosis. This would provide a longer growth period for $T$. gondii in host cells and also benefit the parasite by avoiding elimination of infected cells.

\section{Acknowledgements}

This work was supported by grants from the National Natural Science Foundation of China (No. 81301453), the Laboratory of Parasite and Vector Biology of China, MOPH (No. WSBKTKT201302), the China Postdoctoral Science Special Foundation (No. 2015T80518), the China Postdoctoral Science Foundation (No. 2014M561598), Jiangsu Postdoctoral Science Foundation (No. 1402171C), and the Senior Talent Studying Initial Funding of Jiangsu University (No. 13JDG023, 13JDG127).

\section{Conflict of interest}

The author declares no conflict of interest.

\section{References}

1. Pereira-Chioccola VL, Vidal JE, Su C. Toxoplasma gondii infection and cerebral toxoplasmosis in HIV-infected patients. Future Microbiol. 2009;4(10):1363-1379.

2. Feustel SM, Meissner M, Liesenfeld O. Toxoplasma gondii and the blood-brain barrier. Virulence. 2012;3(2):182-192.

3. Van de Veerdonk FL, Kullberg BJ, Netea MG. Pathogenesis of invasive candidiasis. Curr Opin Crit Care. 2010;16(5):453-459.

4. Luder CG, Campos-Salinas J, Gonzalez-Rey E, et al. Impact of protozoan cell death on parasite-host interactions and pathogenesis. Parasit Vectors. 2010;3:116.

5. Treeck M, Sanders JL, Elias JE, et al. The phosphoproteomes of Plasmodium falciparum and Toxoplasma gondii reveal unusual adaptations within and beyond the parasites' boundaries. Cell Host Microbe. 2011;10(4):410-419.

6. Bradley PJ, Sibley LD. Rhoptries: an arsenal of secreted virulence factors. Curr Opin Microbiol. 2007;10(6):582-587.

7. Wu L, Chen SX, Jiang XG, et al. Separation and purification of Toxoplasma gondii tachyzoites from in vitro and in vivo culture systems. Exp Parasitol. 2012;130(1):91-94.

8. Cleveland DW, Fischer SG, Kirschner MW, et al. Peptide mapping by limited proteolysis in sodium dodecyl sulfate and analysis by gel electrophoresis. J Biol Chem. 1977;252(3):1102-1106.

9. Laliberte J, Carruthers VB. Host cell manipulation by the human pathogen Toxoplasma gondii. Cell Mol Life Sci. 2008;65(12):1900-1915. 
10. Sinai AP, Roepe PD. Autophagy in Apicomplexa: a life sustaining death mechanism? Trends Parasitol. 2012;28(9):358-364.

11. Lim DC, Cooke BM, Doerig C, et al. Toxoplasma and Plasmodium protein kinases: roles in invasion and host cell remodelling, Int $J$ Parasitol. 2012;42(1):21-32.

12. Nelson MM, Jones AR, Carmen JC, et al. Modulation of the host cell proteome by the intracellular apicomplexan parasite Toxoplasma gondii. Infect Immun. 2008;76(2):828-844.

13. Randow F, Munz C. Autophagy in the regulation of pathogen replication and adaptive immunity. Trends Immunol. 2012;33(10):475-487.

14. Hood MI, Skaar EP. Nutritional immunity: transition metals at the pathogen-host interface. Nat Rev Microbiol. 2012;10(8):525-537.

15. Hohl TM, Feldmesser M. Aspergillus fumigatus: principles of pathogenesis and host defense. Eukaryot Cell. 2007;6(11):1953-1963.

16. Shen J, Chen YT, Wu L, et al. Effect of Toxoplasma gondii tachyzoite infection on HeLa cell apoptosis. J Jiangsu Univ. 2014;24(5):399-402.

17. Paredes-Santos TC, de Souza W, Attias M. Dynamics and 3D organization of secretory organelles of Toxoplasma gondii. J Struct Biol. $2012 ; 177(2): 420-430$

18. Nadipuram SM, Kim EW, Vashisht AA, et al. In vivo biotinylation of the Toxoplasma parasitophorous vacuole reveals novel dense granule proteins important for parasite growth and pathogenesis. MBio. 2016;7(4):e00808-e00816.
19. Sinai AP, Joiner KA. The Toxoplasma gondii protein ROP2 mediates host organelle association with the parasitophorous vacuole membrane. $J$ Cell Biol. 2001;154(1):95-108.

20. Jensen $\mathrm{KD}, \mathrm{Hu} \mathrm{K}$, Whitmarsh RJ, et al. Toxoplasma gondii rhoptry 16 kinase promotes host resistance to oral infection and intestinal inflammation only in the context of the dense granule protein GRA15. Infect Immun. 2013;81(6):2156-2167.

21. Cheng L, Chen Y, Chen L, et al. Interactions between the ROP18 kinase and host cell proteins that aid in the parasitism of Toxoplasma gondii. Acta Trop. 2012;122(3):255-260.

22. Kemp LE, Yamamoto M, Soldati-Favre D Subversion of host cellular functions by the apicomplexan parasites. FEMS Microbiol Rev. 2013;37(4):607-631.

23. Schneider AG, Abi Abdallah DS, Butcher BA, et al. Toxoplasma gondii triggers phosphorylation and nuclear translocation of dendritic cell STAT1 while simultaneously blocking IFNgamma-induced STAT1 transcriptional activity. PLoS One. 2013;8(3):e60215.

24. Asai T, Tena G, Plotnikova J, et al. MAP kinase signalling cascade in Arabidopsis innate immunity. Nature. 2002;415(6875):977-983.

25. Nam HW. GRA proteins of Toxoplasma gondii: maintenance of hostparasite interactions across the parasitophorous vacuolar membrane. Korean J Parasitol. 2009;47:S29-S37.

26. D'Angelillo A, De Luna E, Romano S, et al. Toxoplasma gondii Dense Granule Antigen 1 stimulates apoptosis of monocytes through autocrine TGF-beta signaling. Apoptosis. 2011;16(6):551-562. 